\title{
Compression of the optic chiasm due to a lymphoreticular malignancy
}

\author{
R.S. Howard, A.S. Duncombe, C. Owens and E. Graham. \\ The Department of Medical Ophthalmology, St. Thomas' Hospital, London SE1 7EH, UK.
}

\begin{abstract}
Summary: A patient is described who presented with visual loss due to infiltration of the optic chiasm by chronic lymphocytic leukaemia. This case demonstrates intracranial infiltration as a primary presentation of chronic lymphocytic leukaemia without lymphoreticular involvement and, to our knowledge, is the first report of a chiasmal syndrome due to this lymphoproliferative disorder.
\end{abstract}

\section{Introduction}

A bitemporal visual field defect is the hallmark of the optic nerve chiasmal syndrome which is commonly caused by mass lesions, either tumours or aneurysms, when the visual loss is the result of either compression or ischaemia. ${ }^{1}$ However, the optic nerves and chiasma lie within the basal cisterns, in close relationship to the leptomeninges and consequently other infiltrative and inflammatory lesions including metastatic carcinoma, ${ }^{2}$ arachnoiditis, ${ }^{3}$ plasmacytoma ${ }^{4}$ and sarcoidosis ${ }^{5}$ may rarely present with the chiasmal syndrome.

We report the case of a 58 year old man with chronic lymphocytic leukaemia (CLL) who presented with biopsy proven infiltration of the optic chiasm. This case, in which prostatic infiltration was the only other manifestation of CLL, demonstrates intracranial infiltration as a presentation of CLL without lymphoreticular involvement.

\section{Case report}

A previously healthy 58 year old engineer developed deterioration of vision in the right eye in July 1985 which progressed over the next 5 months until he was only able to recognize shapes with the right eye. His general health was good except that in September 1985 he had undergone a transurethral prostatectomy because of a short history of hesitancy and urgency. Postoperatively he developed incontinence and required an indwelling urinary catheter.

Correspondence: R.S. Howard M.A., M.R.C.P., Sobell Department of Neurophysiology, Institute of Neurology, The National Hospital, Queen Square, London WCIN 3BG, UK.

Accepted: 30 July 1987
At the time of presentation to the ophthalmologists, in January 1986, systemic examination was unremarkable. However, ocular examination showed the right visual acuity to be counting fingers with absent colour vision and marked inferotemporal field loss. The right optic disc was pale with attenuated vessels. The left eye was normal.

Routine investigations, including a computed tomographic (CT) scan, were normal but the cerebrospinal fluid (CSF) showed elevated protein of $0.8 \mathrm{~g} / \mathrm{l}$, and a white cell count of $4 \times 10^{y} / 1$. The cause of the visual loss was presumed to be ischaemic although the patient was kept under review.

Over the following three months the vision in both eyes deteriorated. On readmission the visual acuity in the right was counting fingers in a small upper nasal field and 6/36 in the left. He now had a temporal hemianopia in the left eye and both discs showed temporal pallor and attenuated vessels (Figure 1). There was no lymphadenopathy or hepatosplenomegaly and the remaining general examination was normal. Investigation at this time showed the white cell count was now elevated at $18.6 \times 10^{9} / 1$ with an absolute lymphocytosis of $12.9 \times 10^{9} / 1$. The Coombs test was negative and blood urea, electrolytes and liver function tests were normal. Thyroid function tests were normal but the serum prolactin was elevated at $1858 \mu \mathrm{U} / \mathrm{ml}$. (normal range: $120-224 \mu \mathrm{U} / \mathrm{ml}$.). Skull $\mathrm{X}$-ray was normal but a CT scan (Figure 2) now showed the presence of a suprasellar mass, continuous with the optic nerve, pressing posteriorly on the clinoid process.

Biopsy of the right optic nerve was undertaken: macroscopically the optic nerves appeared greyish and histological examination showed diffuse infiltration by mature lymphocytes. The histology of the prostatic tissue was reviewed and this showed similar findings of 


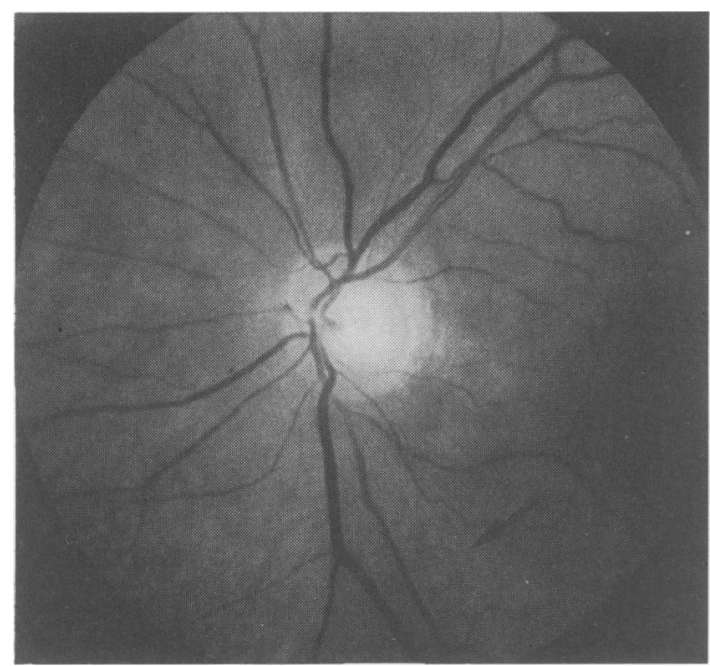

Figure 1 Photograph of left fundi showing attenuated retinal arterioles and easily viewed nerve fibre layer (arrow) indicating loss of axons.

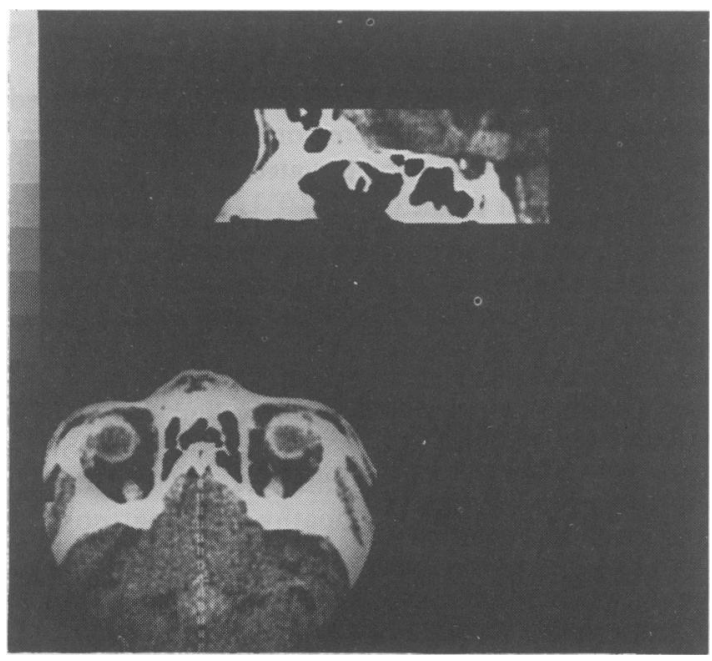

Figure 2 CT scan with saggital reconstruction showing the presence of a suprasellar mass, continuous with the optic nerve, pressing posteriorly on the clinoid process.

pronounced perivascular cuffing by mature lymphocytes.

A bone marrow aspirate showed increased numbers of mature small round lymphocytes, which amounted to $55 \%$ of the total nucleated marrow cells. A high mouse rosette score was obtained and cell surface marker studies were compatible with chronic lymphocytic leukaemia. A repeat lumbar puncture $\stackrel{\mathbb{Q}}{\triangle}$ showed a white cell count of $\mathbf{4 0}$ cells/1, cytospin film $\subseteq$ showing these to be lymphocytes with morphology as $\vec{F}$ in the peripheral blood and bone marrow. CT scans of the chest and abdomen showed no evidence of lymphadenopathy and liver and spleen size were normal.

A diagnosis of chronic lymphocytic leukaemia was made and he received a course of total cranial irradiation together with intrathecal methotrexate of followed by monthly courses of cyclophosphamide, $\overrightarrow{0}$ adriamycin, vincristine, and prednisolone (CHOP) in standard doses.

Repeat CT scan after three months of therapy showed regression of the suprasellar mass, and the CSF and peripheral blood lymphocyte counts have returned to normal. Visual function remains unchanged.

\section{Discussion}

In patients with leukaemia direct involvement of the pituitary gland may occur as the result of infiltration by tumour, haemorrhage, coagulative necrosis, or iatrogenically due to surgery or radiotherapy. ${ }^{6}$ The most common clinical manifestation is infiltration of $\mathbb{\Phi}$ the posterior lobe of the pituitary gland leading to diabetes insipidus. ${ }^{7,8}$ There was no evidence of $\infty$ endocrine disorder in the present patient and the. elevated prolactin may be explained by direct compression of the pituitary gland.

Leukaemia may produce swelling of the optic discs, either by direct infiltration or intracranial haemorrhage resulting in obstruction to CSF flow and raised intracranial pressure. Infiltration of the retrolaminar portion of the optic nerve has been reported in association with multiple myeloma ${ }^{9}$ and solitary plasmacytoma. ${ }^{4}$

Clinically manifest intracranial disease in chronic lymphocytic leukaemia (CLL) is uncommon and is usually due to complicating infection or haemorrhage. Hanson ${ }^{10}$ showed that intracranial lymphocytic infiltration was a frequent autopsy finding but none of the patients had clinical manifestations. Leukaemic meningitis rarely occurs in CLL" although it is relatively common in the acute leukaemias. In the case discussed by Singh \& Thompson", leukaemic meningitis was a late manifestation in a patient who had previously presented with an extensive tissue load of CLL.

This patient is most unusual because chronic lymphocytic leukaemia rarely manifests itself clinically as intracranial disease and has not previously been shown to produce chiasmal compression. Although the patient was only in his late 50 s, he had a stormy post-operative period following his prostatectomy. Pathological studies show that CLL involves the 
prostate gland in the majority of men with the disease. ${ }^{10}$ Hence, perhaps this history should have alerted us to suspect an unusual cause of his bilateral visual loss.

\section{References}

1. Glaser, J.S. In Neuro-ophthalmology, 1st ed. Harper and Row, Haggerstown, Maryland, 1978, pp 140-149.

2. Cohen, M.M., Lessell, S. Chiasmal syndrome due to metastases. Arch Neurol 1979, 36: 565-567.

3. Coyle, J.T. Chiasmatic arachnoiditis. Am J Ophthalmol 169, 68: 345-349.

4. Poon, M.C., Prachal, J.T., Murad, T.M. \& Galbraith, J.G. Multiple myeloma masquerading as chromophobe adenoma. Cancer 1979, 43: 1513-1516.

5. Walsh, T.J. \& Smith, J.L. Sarcoidosis and suprasellar mass. In Smith, J.L. (ed) Neuro-ophthalmology Symposium, Vol. 4. C.V. Mosby, St. Louis, 1968, pp 167-177.

6. Borsey. D.O. \& Sharp. R.A. Hypopituitarism associated

\section{Acknowledgements}

The authors would like to thank Dr R. Ross Russell and Professor L. Symon for permission to publish details of this patient under their care.

with leukaemia. Hospital Update 1986; March: 171-184.

7. Miller, V.I. \& Campbell, W.G. Diabetes insipidus as a complication of leukaemia. Cancer 1971, 28: 666-673.

8. Philippakos, D., Kakouros, S., Dervenoulas, J. et al. Diabetes insipidus as a complication of acute monocytic leukaemia. Postgrad Med J 1983, 59: 93-94.

9. Goudas, P.P. Optic nerve myeloma. Am J Ophthalmol 1971, 71: 1085-1086.

10. Hanson, M.M. Chronic lymphocytic leukaemia. Scand J Haematol 1970, 18: 9-23.

11. Singh, A.K. \& Thompson, R.P.H. Leukaemic meningitis in chronic lymphocytic leukaemia. Acta Haematol 1986, 75: $113-115$. 